\title{
EL PRODUCTOR EJECUTIVO DE PROGRAMAS DE ENTRETENIMIENTO. JERARQUÍAS EN LA PRODUCCIÓN AUDIOVISUAL
}

\author{
Enrique Guerrero \\ (Universidad de Navarra) \\ eguerrero@unav.es
}

\begin{abstract}
Resumen: El presente artículo tiene por objeto analizar el perfil profesional del productor ejecutivo en la industria audiovisual, como máximo responsable creativo y económico de los proyectos de entretenimiento. Para ello, en primer lugar, se confrontan las visiones que sobre esta figura predominan en el sector televisivo anglosajón y en el español y, a continuación, se destacan sus cualidades y aptitudes. Para concluir, se estudian las jerarquías de producción establecidas en las cadenas y productoras de contenidos, así como las funciones de los distintos profesionales que las integran. Como resultado, se obtiene el proceso seguido en la toma de decisiones de toda producción audiovisual.
\end{abstract}

Palabras claves: Productor ejecutivo, producción audiovisual, programas de entretenimiento, televisión.

Abstract: The analysis in this article centres on the professional profile of the executive producer in the audiovisual industry, who is the major creative and financial responsible for the entertainment projects. Firstly, the predominant views about this professional in the Anglo-saxon and in the Spanish television are compared and, secondly, its qualities and its flairs are highlighted. Finally, we study the production hierarchies established in networks and production companies, and the professional categories involved with its functions. As a result, we obtain the followed roadmap in the process of making decisions in every audiovisual production.

Key Words: Executive producer, audiovisual production, entertainment TV shows, television.

\section{INTRODUCCIÓN}

esde los inicios de la televisión en España en los años cincuenta, siempre ha existido una figura creativa central en torno a la que se ha organizado el proceso de producción de programas. Durante las primeras décadas, en Televisión Española (TVE), el realizador-autor destacó como el máximo responsable, asumiendo las tareas de dirección. La escasa profesionalización de la actividad y la no consideración del sector como una industria situaron a un profesional de un perfil exclusivamente artístico-creativo como eje de todo el proceso. 
El productor ejecutivo de programas de entretenimiento. Jerarquías

en la producción audiovisual

La separación de funciones y la distinción del realizador y del director como dos profesionales independientes tuvo su origen a mediados de los 60 cuando se puso en marcha el segundo canal de TVE. Por su perfil cultural y la incorporación a su plantilla de personal procedente de la cinematografía, que desconocía las posibilidades de las cámaras electrónicas, muchos directores necesitaron de realizadores con experiencia previa en televisión. Este desdoblamiento no llegó a producirse en el mundo anglosajón, donde el director asume la doble tarea de dirigir tanto el contenido como la puesta en escena y en imagen.

No fue hasta la configuración de la producción televisiva como industria y como negocio, cuando se comenzó a considerar al productor ejecutivo como el máximo responsable de los proyectos, tanto desde una perspectiva económica y logística, como creativa, realidad habitual en la práctica profesional anglosajona (Guerrero, 2010: 376). A su vez, la consideración de la actividad audiovisual como industria llevaba implícita una visión del contenido de entretenimiento producido como producto, aunque con las características peculiares de todo bien cultural (Herrero y Diego, 2009; Luengo, 2008).

Tradicionalmente, en Europa, en el sector televisivo, al igual que ha ocurrido en otras industrias audiovisuales como la cinematográfica, se ha prestado una mayor atención a las figuras del director y del realizador en detrimento de la del productor. Esta circunstancia ha quedado reflejada en la literatura académica publicada sobre producción audiovisual, más centrada en la realización y en la dirección que en la figura del productor. En este sentido, destacan autores como Millerson (1961, 1983, 1991, 2009), De Aguilera (1965), Newcomb y Alley (1983), Sáinz (1990, 1995, 1999), Fernández y Martínez (1994), Hart (1999), Bustamante (1999), Barroso (2002), Pardo (2002), Diego (2005), Zettl (2006) y Kellison (2009) entre otros.

Sin embargo, en televisión, el papel del productor es de vital importancia, pues sobre él recae en último término la responsabilidad asociada al desarrollo y al control de la producción del proyecto, no sólo desde un punto de vista económico y logístico, sino también creativo (Diego González, 2005: 9-30). La hipótesis de partida, por tanto, sostiene que el productor ejecutivo de contenidos de entretenimiento no es un mero burócrata, sino un gestor creativo que debe asumir el liderazgo y tener las cualidades adecuadas para ejercer sus funciones con autoridad y, a la vez, coordinar el trabajo en equipo. Aún más, si se toma en consideración el contexto multiplataforma actual en el que se inserta el sector televisivo.

No obstante, conviene advertir de que, habitualmente, la producción ejecutiva no recae de forma exclusiva en un único individuo, sino que sus responsabilidades se distribuyen, según lo acordado en el contrato de producción, entre los equipos de producción de cadenas y productoras. Además, no siempre existe en las jerarquías de producción la figura específica del productor ejecutivo, dado que esta función puede ser desempeñada por un alto dirigente del canal o de la empresa productora. En cualquier caso, sus funciones siempre deben ser asumidas por uno o varios cargos identificables, independientemente de que quien las ejerza sea un productor ejecutivo perteneciente a la estructura de la empresa o 
un puesto directivo con más responsabilidades.

Asimismo, hay que distinguir varios niveles en la toma de decisiones referidas al proceso de producción. Por un lado, la producción ejecutiva, que asume las decisiones más relevantes, incluida la puesta en marcha o no de un proyecto; y por otro, la producción de campo, cuyas competencias se centran en la realización concreta del programa.

Todo ello queda reflejado en la estructura del presente artículo, cuyo objeto es delimitar las actividades que competen al área de la producción televisiva y de su principal protagonista, el productor ejecutivo. En primer lugar, analizaremos la figura del productor de televisión y sus cualidades más características. A continuación, nos detendremos en las jerarquías de los distintos equipos de producción de cadenas y productoras para, finalmente, concretar los diferentes cargos que las componen, analizar sus funciones y dibujar la ruta seguida en la toma de decisiones.

\section{METODOLOGÍA}

Dado el carácter académico y profesional de la investigación en la que se basa este artículo, además de consultar la bibliografía especializada sobre producción ya detallada más arriba, ha sido imprescindible contar con la colaboración de profesionales del sector audiovisual -productores ejecutivos en su mayoría-. Se han involucrado mediante la concesión de entrevistas semi-estructuradas y la respuesta a detallados cuestionarios compuestos por unas 80 preguntas, cuyos resultados han permitido reconstruir -etapa a etapa- el proceso de toma de decisiones en la producción audiovisual y el perfil de su máximo responsable, el productor ejecutivo. Debido a la dualidad de protagonistas que participan en la industria televisiva -cadenas y productoras-, se ha optado por no emplear un único modelo para todos los profesionales, sino que se han diseñado entrevistas y cuestionarios específicos para los siguientes perfiles: productor ejecutivo de cadena y productor ejecutivo de productora.

En este trabajo han participado los principales agentes de la industria televisiva española, considerando factores como su volumen de producción, su trayectoria o el éxito de sus contenidos. Entre las empresas que han colaborado, como se detalla al final del presente artículo, destacan Televisión Española (TVE), Tele 5, Antena 3, Gestmusic-Endemol, Globomedia, Cuarzo, Grundy, El Terrat, Europroducciones, Martingala TV, Videomedia, Notro TV y 7 y Acción.

El apoyo de la industria ha resultado crucial para poder realizar este estudio, dada la menor atención que se le ha prestado a la figura del productor desde la academia, más atenta a otros perfiles profesionales del audiovisual como el director o el realizador. Esta es la razón por la cual el presente artículo pretende realizar una reflexión en torno a la figura del productor, destacando sus cualidades y sus funciones en el proceso de producción audiovisual, comparando la visión anglosajona y la española.

\section{EL PRODUCTOR EJECUTIVO DE PROGRAMAS DE ENTRETENIMIENTO}


El productor ejecutivo de programas de entretenimiento. Jerarquías en la producción audiovisual

De modo genérico, emplearemos el término productor para referirnos al responsable máximo del desarrollo de un proyecto y de su posterior producción, es decir, a aquel profesional implicado desde el inicio en la creación, realización y comercialización de un programa. Por tanto, nos ceñiremos, de momento, a la producción ejecutiva, y no a la de campo. Desde esta perspectiva, dos son sus principales facetas: actuar como director de proyectos (project manager) y tomar decisiones cruciales tanto empresariales como creativas (Pardo, 2002: 49).

Antes de adentrarnos en el análisis de sus cualidades y funciones, vamos a delimitar el concepto de productor ejecutivo, figura sobre la que no existe un consenso en la industria de modo global y cuyas atribuciones varían de un mercado a otro. Ello se refleja en las diferentes visiones que sobre este profesional predominan en España y en los países anglosajones.

\subsection{El perfil español frente al anglosajón}

Tanto en la industria española como en la anglosajona, el productor ejecutivo destaca como el máximo responsable del proyecto. Sin embargo, existen diferencias respecto al perfil de esta figura en ambos casos, especialmente, referidas a su autoridad en la toma de decisiones de orden creativo. Para abordar este debate, haremos referencia en primer lugar a los contextos legales de ambos mercados, dado que, a nuestro entender, reflejan la esencia de los distintos perfiles ocultos tras ambas visiones.

Respecto a la industria televisiva española, el Libro Blanco del Audiovisual define la figura del productor como "la persona física o jurídica, bajo cuya iniciativa y responsabilidad se lleva a cabo la obra audiovisual" (Écija Bernal, 2000: 44). En este sentido, el texto legal que sirve de referencia es la Ley 23/2006, de 7 de julio, de propiedad intelectual (LPI), de la que parte la definición anterior, y en donde se especifica que el productor es aquella "persona natural o jurídica que tenga la iniciativa y asuma la responsabilidad de dicha grabación audiovisual" (art. 120). No obstante, a pesar de ser considerado promotor y máximo responsable de la producción, no ostenta la categoría de autor. Como es bien sabido, según la legislación española, tienen consideración de autor de la obra audiovisual el director-realizador y los autores del argumento, de la adaptación, del guión, de los diálogos y de las composiciones musicales (art. 87). A todos ellos, hay que añadir al director de fotografía en el caso de las obras cinematográficas según la Ley 55/2007, de 28 de diciembre, del cine (art. 5).

Sin embargo, la LPI sí reconoce al productor unos derechos de propiedad sobre la obra, que le son cedidos en exclusiva por los autores, mediante la firma del contrato de producción. Así lo recoge el artículo 88 de la citada ley: "Sin perjuicio de los derechos que corresponden a los autores, por el contrato de producción de la obra audiovisual se presumirán cedidos en exclusiva al productor, con las limitaciones establecidas en este Título, los derechos de reproducción, distribución y comunicación pública, así como los de doblaje o subtitulado de la obra".

La cesión de estos derechos no implica la renuncia de los autores a una remuneración, que deberá determinarse para cada una de las modalidades de explotación (art. 90). Además, no todos los derechos se presumen cedidos, como 
es el caso del derecho de transformación o sobre los productos derivados (merchandising), así que todo ello debe ser recogido de forma explícita y detallada en el contrato de producción (Écija Bernal, 2000: 45).

Como resultado, y aunque la ley no lo registre como tal, el productor ejecutivo asume, en cierto modo, el papel de un autor en la sombra, debido a la impronta personal que lleva cada uno de los proyectos de los que es el máximo responsable. Así lo expresa Barroso: "De este panorama surge la figura del productor ejecutivo como la del autor oculto, ya que, de algún modo, el sistema de producción en cadena, despersonalizado, fragmentado, exige una figura coordinadora, responsable del producto final y capaz de imprimir una huella o marca propia y peculiar" (2002: 36).

A diferencia de la legislación latina, la anglosajona concede la categoría de autor al productor, tal como queda recogido en El Libro Blanco del Audiovisual: "Como ya se ha señalado, en el modelo anglosajón el productor es el titular originario de los derechos y tiene plena libertad para la explotación de los mismos, sin perjuicio de los derechos de remuneración que pudieran corresponder a aquellos que hubieran realizado las aportaciones creativas de la obra" (Écija Bernal, 2000: 45).

Estas diferentes visiones del productor van más allá de una mera consideración legal, y tienen consecuencias directas en la configuración de la industria de la producción audiovisual y en el papel desempeñado por la figura del productor ejecutivo. En el caso anglosajón, predomina una concepción del sector como industria en la que las decisiones cruciales son tomadas por los productores ejecutivos, de quienes depende la puesta en marcha y el desarrollo de los proyectos, y cuyo perfil es empresarial y creativo al mismo tiempo.

Newcomb y Alley conceden a este profesional la máxima autoridad del equipo creativo: "The producer is the person in charge of a TV production, who stablishes the working spirit and dictates the standards to be met. Ideally, as head of the creative team, the producer is both businessman and artist, caring about administration and budgetary details while nurturing the talent and providing the vision for the Project" (1983: 8)2.

En esta misma línea, Blumenthal y Goodenough consideran que "the executive producer usually supervises all creative aspects of the program", y también destacan su doble perfil empresarial y artístico: "Executive producer refers to the business person most responsible for getting the series on the air -or arranging

\footnotetext{
${ }^{1}$ Para conocer más acerca de los derechos de autor de la obra audiovisual, véase AZURMENDI ADARRAGA, A. (2011): Derecho de la comunicación. Barcelona: Bosch.

${ }^{2}$ Traducción: "El productor [de televisión] es aquella persona encargada de una producción televisiva que establece el espíritu de trabajo y dicta los estándares que deben cumplirse. En una situación ideal, como cabeza del equipo creativo, el productor es al mismo tiempo un empresario y un artista, pendiente tanto de detalles administrativos y presupuestarios como de cuidar el talento y de dotar de una visión al proyecto".
} 
El productor ejecutivo de programas de entretenimiento. Jerarquías

en la producción audiovisual

the majority of the production financing- or to the senior creative person on the Project" (1991: 229) ${ }^{3}$.

En nuestro entorno, la figura más próxima al executive producer americano, industria de referencia en el ámbito del entretenimiento audiovisual, es la representada por el productor ejecutivo. Sin embargo, como advierte Joaquim Amat, director de producción de Televisión de Cataluña (TVC), "Ios modelos nunca son directamente aplicables" (Fernández Diez y Martínez Abadía, 1994: 9). Así pues, en su traslación a nuestro sistema, la industria española dio origen a un productor ejecutivo cuyas funciones reales se limitaban a cuestiones organizativas y económicas, dejando de lado la faceta más relevante del producer estadounidense: la creativa.

En este sentido, Fernández y Martínez advierten de la desnaturalización sufrida por la figura del productor ejecutivo en España: "Los productores, personas capacitadas para dirigir, controlar y gestionar los proyectos audiovisuales en sus vertientes creativa, organizativa y ejecutiva, con distintos grados de responsabilidad, indispensables para llevar a buen puerto los filmes cinematográficos, producciones de vídeo y programas de televisión, han sido considerados muchas veces meros burócratas, elementos financieros alejados de la "autoría" de la obra, ejecutivos puestos por la empresa para constreñir la creatividad de los directores y guionistas" (1994: 11).

Este mismo argumento también es esgrimido por Contreras y Palacio: "En Estados Unidos se trata mayoritariamente de un creativo al que se le asigna el control sobre el proyecto. Se trata así de un auténtico responsable directo de los aspectos artísticos, nunca de un ejecutivo de la cadena encargado de la supervisión o de un responsable del control de los recursos de producción" (2001: 150).

No obstante, Fernández y Martínez apuntaban ya a mediados de los 90 que "los productores comienzan a ser considerados parte fundamental del proceso creativo" (1994: 11). Muestra de ello es que la mayoría de los productores ejecutivos entrevistados para esta investigación, en el caso de las productoras, son a la vez los máximos responsables del área de producción de programas de entretenimiento. Además, coinciden en afirmar que tienen poder de decisión sobre el contenido del programa junto a otros profesionales (cuestionario realizado a productores ejecutivos de productoras y cadenas españolas, 2008):

1. Por parte de la productora: el responsable del área de producción de programas de entretenimiento, el productor ejecutivo -en el caso de que sea

\footnotetext{
${ }^{3}$ Traducción: "El productor ejecutivo normalmente supervisa todos los aspectos creativos del programa". "El productor ejecutivo es el empresario responsable de la emisión del programa -o de conseguir la mayor parte de la financiación necesaria para la producción- o el máximo responsable creativo del proyecto". Para conocer más acerca del producer en la industria anglosajona, véase SCHIHL, R. J. (1992): Talk show and entertainment program: processes and procedures. Boston: Focal Press; MILLERSON, G. (1993): Effective TV production, Londres: Focal Press; HART, C. (1999): Television program making: everything you need to know to get started. Oxford: Focal Press; CLEMENTS, S. (2004): Show Runner: Producing Variety and Talk Shows for Television. Los Angeles: Silman-James Press; y ZETTL, H. (2006): Television Production Handbook. Belmont: Thomson Wadsworth
} 
una persona distinta a quien ostente el cargo anterior- y el director del programa.

2. Por parte de la cadena: el director de la división de producción general de programas y el productor ejecutivo.

En consecuencia, Fernández y Martínez consideran que la figura del productor ejecutivo creativo, importada de la industria estadounidense, cada vez tiene una mayor presencia en España: "La idea de un productor ejecutivo a la americana, que vendría a convertirse en un director de proyectos (uno o varios de los que es el máximo responsable), se está encarnando paulatinamente en Europa en la ya existente figura del jefe de área o departamento que, cada día más, se implica en la dirección, seguimiento y control de los proyectos" (1994: 83).

A continuación, vamos a centrarnos en las cualidades que debe poseer el productor ejecutivo de contenidos de entretenimiento en cuanto que responsable máximo de los proyectos, tanto en su vertiente empresarial como creativa, según los principales autores y los profesionales del sector consultados.

\subsection{Cualidades del productor ejecutivo}

Aunque este apartado se centre en un único protagonista, lo cierto es que la producción audiovisual se considera, fundamentalmente, un trabajo en equipo cuyo liderazgo es asumido por el productor ejecutivo. Para ello, cabe esperar de él una serie de aptitudes que, según Sáinz, pueden resumirse en las siguientes (1999: 20):

1. Comprensión, reflejos, empatía, arrastre.

2. Formulación de la política general de la empresa.

3. Vigilancia de los programas de acción: coordinación y supervisión.

4. Determinación del organigrama ideal.

5. Conocimientos técnicos.

Existe un consenso general respecto a considerar la figura del productor ejecutivo como un líder a cargo de un equipo y al frente de un proyecto del cual él es el máximo responsable. En palabras de Sáinz, "es responsable del resultado artístico y técnico" y "quien promueve e idea los proyectos" (1995: 17 y 23). Por tanto, la capacidad de liderazgo sobresale como una de sus características principales, tal y como también lo indica Joaquim Amat, según su experiencia en la televisión autonómica catalana: "La creación de un producto audiovisual es el resultado de un trabajo en equipo. Equipo multidisciplinario que necesita de un líder, con autoridad, responsabilidad y talento para planear las operaciones y tomar decisiones complejas" (Fernández Díez y Martínez Abadía, 1994: 9). 
Además, y a pesar de ser considerado en muchos ámbitos un mero burócrata dedicado a cuestiones económicas y organizativas, la mayor parte de las fuentes consultadas coinciden en atribuir la condición de creativo al productor ejecutivo. Víctor Martín, productor ejecutivo de programas de entretenimiento de Globomedia, incide en este aspecto: "Tiene que ser creativo, porque es él quien define la línea de los programas. Para ello, debe tener muy claro el concepto del formato que quiere hacer y exprimirlo para sacarle toda la calidad posible. Tiene que entender el formato y cómo hay que adaptarlo en España. El trabajo de productor ejecutivo es como ser capitán de un barco, debe saber qué rumbo tomar en cada momento según la dirección del viento" (entrevista a Víctor Martín, 16/06/08).

Según Daniel Bilbao, presidente de Martingala TV y productor ejecutivo de programas de entretenimiento, "un buen productor ejecutivo debe saber de contenidos y, después, de producción" (entrevista a Daniel Bilbao, 17/06/08). En esta misma línea, José Jesús Lara, director de la división de producciones autonómicas y productor ejecutivo de Europroducciones, afirma que, en su caso, posee un "control presupuestario, logístico y en contenidos" sobre los programas bajo su supervisión (entrevista a José Jesús Lara, 16/06/08).

Además de cualidades creativas y capacidad de liderazgo, el productor ejecutivo debe ser polivalente y poseer amplios conocimientos relacionados con las distintas áreas de la industria televisiva. Sáinz considera que la especialización en determinados contenidos no exime de la obligación de conocer todas aquellas herramientas que puedan hacer más eficiente la producción de cualquier género televisivo: "La producción de programas de televisión supone para el productor audiovisual el objetivo de su trabajo. La variedad de productos es un reto de planificación que entraña la responsabilidad de conocer todas las variantes posibles. Aunque puede existir especialización en un determinado género de programas, el productor audiovisual debe disponer de un conocimiento pormenorizado de todas las 'áreas del negocio audiovisual', y esto supone conocer las herramientas y medios que pueden serle útiles para una gestión eficaz" (1999: 22).

Esta cualidad es aún más relevante en el contexto multiplataforma actual de la televisión, dado que también es competencia del productor ejecutivo la supervisión y coordinación de la estrategia multimedia e interactiva de los contenidos de entretenimiento en todas las pantallas, según Jorge Ventosa, director de producción de 7 y Acción (entrevista a Jorge Ventosa, 25/11/11).

No obstante, no basta con poseer conocimientos, sino también es necesario contar con la experiencia suficiente como para haberlos puesto en práctica. Víctor Martín se muestra contrario al intrusismo profesional en el campo de la producción televisiva, y aboga por la formación de los productores ejecutivos en el propio sector: "Un productor ejecutivo de programas tiene que proceder de la industria de la televisión. En algunos casos, gente con dinero amplia su negocio y se mete en la producción. Pueden tener suerte una vez, pero no más. El productor 
ejecutivo es necesario que sepa de producción, de realización y de técnica. $\mathrm{Si}$ sabe de todo eso, sabe de dinero" (entrevista a Víctor Martín, 16/06/08).

En este mismo aspecto incide Daniel Acuña, director de la división de programas de entretenimiento de Videomedia, para quien la experiencia en la profesión aporta credibilidad: "El productor ejecutivo debe haber trabajado mucho en esta profesión, en muchos y variados programas. Hacer de todo aporta credibilidad. No tiene por qué saber absolutamente de todo, pero sí al menos tener algún conocimiento sobre realización y contenidos" (entrevista a Daniel Acuña, 13/06/08).

Además de todo ello, según Ramón González, ex-productor ejecutivo de Tele 5 y ex-subdirector de programas de Telemadrid, al productor ejecutivo debe gustarle la televisión y tener una especial intuición para conseguir que sus programas destaquen sobre el resto: "Le debe gustar el medio y entender cada programa que hace, conociendo el sentido que le imprimió el creador del formato. Debe ser un profesional con intuición para saber qué es lo que hace interesante a un programa. Aunque la experiencia le aportará el conocimiento técnico, no basta con un programa bien hecho, sino que tiene que saber darle sentido y hacerlo diferente de los demás" (entrevista a Ramón González, 14/06/08).

Por último, mencionaremos unas de las cualidades más importantes: la capacidad de gestión, organización y coordinación. Esta característica va muy ligada al papel del productor ejecutivo como líder de un equipo, en el que necesariamente deberá confiar para poder delegar determinadas tareas, especialmente relevantes en el caso del productor y del director de los programas. Manuel Aguilera, ex-director de producción de programas de entretenimiento de Grundy, sintetiza del siguiente modo estas cualidades: "Un buen productor ejecutivo debe ser capaz de controlar los contenidos del programa, como último responsable que es, coordinar la organización de los equipos y controlar el presupuesto. A esto hay que añadirle la dificultad de que no gestiona el presupuesto ni dirige el programa directamente, sino que debe hacerlo manteniendo una buena relación con el director del programa y con el director de producción o productor, que son como sus dos manos" (entrevista a Manuel Aguilera, 17/06/08).

A pesar de todos los cambios sufridos por la industria televisiva a lo largo de los años, las cualidades esenciales de un buen productor ejecutivo, a día de hoy, son básicamente las mismas que antaño. Ello se confirma si comparamos las características mencionadas unas líneas más arriba con las recogidas en uno de los primeros libros escritos por un autor español sobre la producción televisiva.

En 1965, el Servicio de formación de TVE publicó el libro La realización en televisión, de Joaquín de Aguilera Gamoneda, primer libro sobre la profesión en España, en el que se le atribuyen al productor las siguientes cualidades:

"Su principal función será la de poseer un conocimiento suficiente del medio en que ha de operar. Así, deberá estar familiarizado con todo el material técnico 
El productor ejecutivo de programas de entretenimiento. Jerarquías en la producción audiovisual

de una emisión y conocer las cámaras y sus posibilidades, los objetivos y su utilización [...]. El buen productor deberá saber inspirar confianza a sus colaboradores y tener la autoridad suficiente para ser capaz de conseguir que éstos actúen no como un conjunto de individuos, sino formando este todo homogéneo llamado "equipo". Deberá también tener (o por lo menos aparentar) una gran calma en todo momento y contingencia, no sólo para poder adoptar decisiones sin verse interferido por los nervios, sino también para infundir serenidad y calmar los posibles nerviosismos de sus colaboradores. El productor de televisión tendrá, asimismo, que ser capaz de tomar decisiones instantáneas y de resolver certera y rápidamente cualquier contingencia imprevista [...]. En resumen, las cualidades primordiales de un buen productor de televisión deberán ser la serenidad y autoridad, debiendo poseer al propio tiempo unos nervios de acero y una gran rapidez en reacciones y reflejos mentales, además del sentido artístico, que se da por supuesto" (1965: 26 -27).

En la medida en que las características mencionadas se encuentren presentes en la figura del productor ejecutivo, estará más o menos capacitado para ejercer las funciones que le competen por encontrarse en una de las posiciones más altas de la jerarquía en la toma de decisiones.

\section{JERARQUÍAS EN LA PRODUCCIÓN AUDIOVISUAL}

Como en toda industria, en la televisiva, la toma de decisiones también sigue una jerarquía que se refleja en la estructura organizativa de cadenas y productoras. En primer lugar, abordaremos el modo en el que se organizan las empresas audiovisuales implicadas en la producción y emisión de contenidos; y en segundo término, nos detendremos en cómo se interrelacionan sus equipos en la toma de decisiones respecto a la puesta en marcha de proyectos de programas y en su proceso de producción. Solo después, abordaremos las diferentes funciones de los profesionales que intervienen en esta actividad.

No existe un consenso en el sector sobre la estructura jerárquica más apropiada ni sobre las denominaciones de sus categorías profesionales. Debe interpretarse, por tanto, el modelo descrito a continuación, como una propuesta basada en las fuentes consultadas y, a la vez, como la fórmula más idónea a nuestro entender para lograr la eficiencia en la actividad de la producción televisiva.

Los cambios experimentados durante los últimos años en la industria de la televisión, debido al crecimiento de la oferta y al desarrollo de un entorno multimedia, no sólo han influido en las modalidades de producción y programación y en las relaciones de propiedad entre cadenas y productoras ${ }^{4}$, sino que también han obligado a estas compañías a remodelar sus esquemas de organización y sus cúpulas directivas. Hasta el año 2005, lo habitual era que el organigrama de una empresa de televisión respondiera a las necesidades de gestión de una sola cadena (mono-canal), que constituía el núcleo del negocio, con una estructura 
jerárquica vertical.

Sin embargo, a partir del relanzamiento de la TDT en 2005 y como consecuencia del proceso de convergencia de la televisión con Internet, las empresas de televisión se reconvirtieron en complejos grupos multimedia con una multiplicidad de canales y ventanas de distribución, que requerían una revisión de su estructura organizativa. Entonces, los operadores comenzaron a optar por una estructura horizontal que permitiese gestionar de una forma coordinada todas las plataformas de explotación y aprovechar de un modo más eficiente las sinergias posibles en los procesos de producción y comercialización de los contenidos. A raíz de la remodelación de sus esquemas de funcionamiento y de gobierno, se obtuvo un organigrama estructurado en divisiones paralelas que se correspondían con las diferentes vías de distribución, más un área única dedicada exclusivamente a la producción de contenidos para todas las plataformas, dependientes todas ellas de un mismo núcleo directivo encargado de su coordinación (Guerrero, 2010: 183-184).

Según Sáinz, uno de los principales problemas que ha lastrado la actividad de las empresas de televisión españolas es la centralización en la toma de decisiones, que recae de forma exclusiva sobre los altos directivos, cuya libertad de actuación está además desvinculada de una asunción de responsabilidad similar. Este autor aboga por una descentralización de los procesos de trabajo: "Las funciones del productor ejecutivo requieren de una organización fuertemente descentralizada que choca con la cultura empresarial actual. Para mejorar la gestión podría delegarse la toma de decisiones en aquellas personas que tienen la información, exigiendo responsabilidad sobre los resultados y retribuyendo el puesto en función de los mismos" (1995: 47).

Sáinz reclama una mayor autonomía y más poder para los productores ejecutivos, auténticos responsables creativos y económicos de los programas bajo su control: "El productor ejecutivo debe tener capacidad suficiente para diseñar los contenidos de los programas, libertad en todo el proceso de decisión económica, incluyendo la asignación presupuestaria que estime oportuna para cada producto [...]. El productor ejecutivo está comprometido con la gestión de los recursos humanos y medios técnicos que forman parte de la dotación del área de producción que dirige" (1999: 21).

En cuanto a las empresas de producción de contenidos, la mayoría de las productoras consultadas presentan una división de programas de entretenimiento compuesta por el director del área, que suele ejercer de productor ejecutivo, y un

\footnotetext{
${ }^{4}$ Para profundizar en cómo los cambios acaecidos en la industria televisiva española a mediados de la primera década del siglo XXI afecta a las modalidades de producción audiovisual, véase FERNÁDEZQUIJADA, D. (2009): "El mercado de la producción independiente en España ante la aparición de Cuatro y laSexta". Comunicación y Sociedad, 22, junio, pp. 59-88; y FERNANDEZ-QUIJADA, D. (2009): "Producción independiente de televisión en Andalucía. Aproximación a la estructura de un sector desconocido". Ámbitos, 18, pp. 191-209.
} 
El productor ejecutivo de programas de entretenimiento. Jerarquías

en la producción audiovisual

director de producción centrado en cuestiones económicas (gráfico 1). Además, casi todas ellas cuentan con un departamento de desarrollo de proyectos.

La jerarquía de producción resultante de la interacción de la estructura organizativa de las cadenas de televisión y de las productoras queda reflejada en el siguiente gráfico. Esta organización jerárquica es típica de la producción propia externa o independiente (Bustamante, 1999: 107-109), modalidad predominante en la industria audiovisual española.

Este organigrama representa la modalidad de producción propia externa más compleja, la asociada, en la que la cadena suele aportar el personal y los recursos técnicos, y la productora, aquellos relacionados con los aspectos creativos y artísticos. Cada equipo de producción gestiona los recursos y la parte del presupuesto que le corresponde según lo acordado en el contrato de producción del programa. La modalidad de producción financiada seguiría el mismo esquema que el presentado, con la singularidad de que el equipo de producción de la cadena se vería reducido a la figura del productor, dado que el peso de la producción recaería sobre la productora, limitándose el operador emisor a supervisar el proceso como agente que aporta la financiación (Guerrero, 2010: 200-201).

A continuación, analizaremos la jerarquía de producción mencionada y describiremos las funciones de las principales categorías profesionales sobre las que recae la toma de decisiones.

\subsection{La jerarquía de producción en la empresa de televisión}

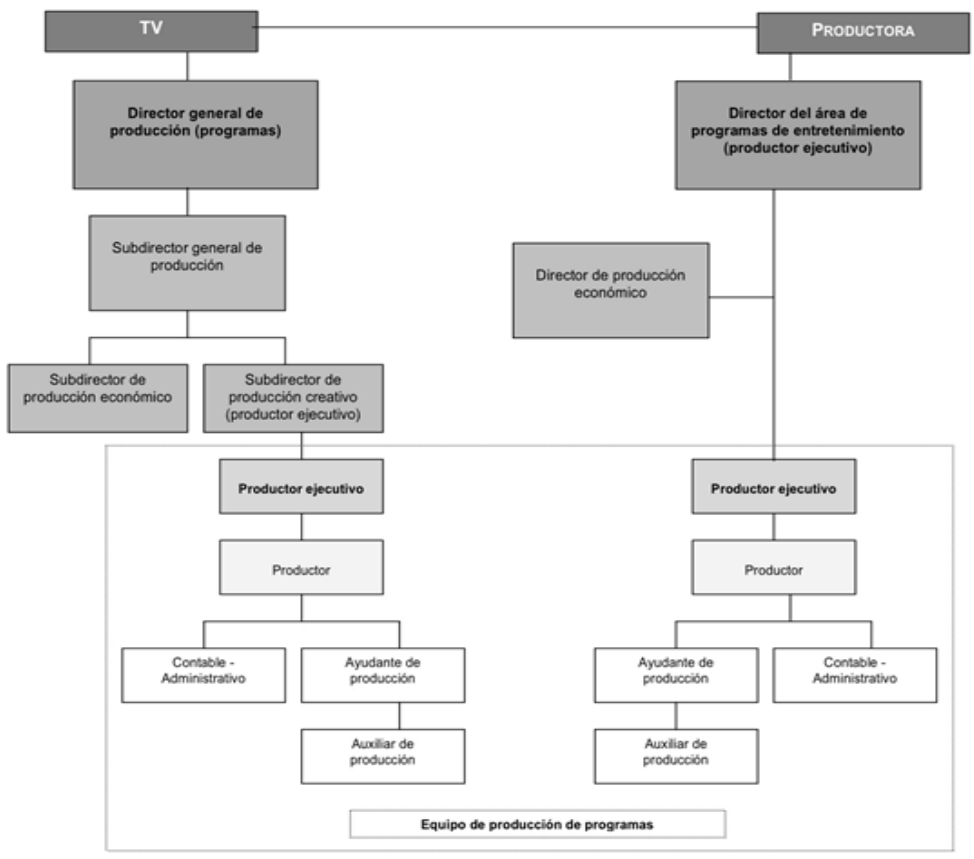


Conviene advertir una vez más que las empresas de televisión españolas no presentan exactamente la misma estructura organizativa. No obstante, es posible encontrar una serie de protagonistas comunes a todas ellas, permitiéndonos realizar las propuestas de organización expuestas más arriba.

Nos ceñiremos en este epígrafe, exclusivamente, al departamento de programas de entretenimiento encuadrado en la división de producción, y cuya estructura jerárquica se corresponde con la apuntada en el gráfico 1. Básicamente, su actividad principal se centra en la creación del contenido, desde la idea originaria hasta su materialización en imágenes (Medina Laverón, 1998: 94). A la hora de describir los perfiles más importantes, seguiremos un orden descendente en la jerarquía de producción, comenzando por los puestos de mayor responsabilidad, y prestando especial atención a aquellos que conforman el equipo de producción de los programas (perfiles enmarcados por una línea discontinua en el gráfico).

El máximo responsable del área de producción de programas de entretenimiento es el director general de producción, apoyado en ocasiones por un subdirector general, que, junto con el director general de contenidos de la empresa de televisión y el director de antena o emisiones, decide qué proyectos se ponen en marcha y de qué forma, cuáles no, y cuándo se cancela la emisión de un espacio. De esta figura depende en última estancia la gestión del presupuesto del área de entretenimiento de la cadena -o grupo de canales-, la firma de los contratos de producción y la supervisión del correcto desarrollo de todo el proceso, tanto desde un punto de vista creativo, como logístico y económico. Desde esta última perspectiva, Fernández y Martínez destacan las siguientes funciones de este directivo: "El director de producción es la persona que establece los presupuestos generales y que concede los medios humanos, técnicos y financieros precisos para efectuar la producción. Establece el marco económico y financiero para producir los programas" (1994: 82).

Para desarrollar su labor, cuenta con un subdirector de producción económico para el conjunto de todos los programas, y con varios subdirectores de producción creativos especializados en determinados tipos de contenidos. Por un lado, el primero de ellos, el económico, controla que los programas en producción respeten el marco presupuestario establecido desde la dirección general de contenidos y sirve de nexo con el departamento de gestión de la empresa televisiva. Y por otro, los directores de producción creativos, que disponen de una cartera de programas pertenecientes a un mismo o a varios géneros, supervisan el tratamiento de los contenidos así como su evolución a lo largo de las emisiones según los resultados de audiencia y su adecuación a la imagen de la cadena. Los directores de producción creativos pueden, a su vez, ejercer de productores ejecutivos, o delegar las tareas más burocráticas de esta función en cargos dedicados exclusivamente a ello ${ }^{5}$.

Según Bardají y Gómez, a diferencia de lo que ocurre en la producción de 
El productor ejecutivo de programas de entretenimiento. Jerarquías en la producción audiovisual

ficción, "en el departamento de programas se produce una mayor separación entre los campos creativo y económico". Estos autores explican que esta circunstancia se debe a que los productores ejecutivos del área supervisan de forma simultánea varios proyectos, y que no siempre deciden implicarse en todas las cuestiones del día a día de la producción (2004: 136).

El productor ejecutivo de una cadena es, por tanto, un directivo bajo cuyo control creativo y económico posee una serie de programas determinados, alzándose como el máximo responsable de la adecuación de medios y fines (Sáinz Sánchez, 1999: 20). Según Sáinz, sus funciones pueden resumirse en los siguientes puntos (1995: 46):

1. Se encarga de proponer y desarrollar ideas de programas dentro de un área específica de programación.

2. Gestiona tanto los equipos humanos como técnicos, distribuyendo estos recursos entre diferentes proyectos de producción.

3. Busca financiación, coopera con instituciones y entidades públicas y privadas con el objeto de resolver los problemas económicos y de contenido que se presenten durante la producción.

4. Establece una distribución racional del presupuesto de su área entre los programas que están a su cargo.

5. Controla que la cantidad y la calidad de producto sea la prevista de acuerdo a planes estratégicos de programación.

6. Programa la emisión (dentro de los márgenes asignados) de sus productos, para lo cual tiene en ocasiones ciertas atribuciones sobre la rejilla de programas.

7. Comercializa tanto la publicidad como el posible patrocinio de los programas que produce, y otros subproductos como mascotas, logotipos, músicas, etcétera.

En esta misma dirección apuntan Fernández y Martínez al describir el perfil del productor ejecutivo:

${ }^{5}$ En la práctica profesional, estos cargos se denominan, simplemente, subdirectores de producción, sin los adjetivos de "económico" y "creativo". No obstante, hemos optado por añadirlos para especificar que pueden especializarse en aspectos creativos o económicos de la producción. 
El jefe de producción, productor ejecutivo o productor jefe es, en una televisión, el responsable de las distintas áreas o departamentos en que se estructuran estas corporaciones y es quien asigna el productor a cada programa. Su actividad gira en torno a la búsqueda, invención y confección de ideas para proyectos audiovisuales. Gestiona el personal operativo principal, elige al director y realizador de cada programa y supervisa varios programas o series a la vez, desde el punto de vista artístico y financiero (1994: 82).

Para Ramón González, según su experiencia en Telecinco y Telemadrid, el productor ejecutivo de la cadena ejerce, principalmente, una función supervisora: "El productor ejecutivo tiene que controlar que se vayan cumpliendo los objetivos marcados por ambas partes, que la productora realmente ponga los recursos por los que la cadena paga. Comprueba que el programa se graba en el tiempo pactado y con los recursos acordados". Además, González también incide en la responsabilidad de esta figura en un doble plano, el logístico y el creativo: "El productor ejecutivo es el responsable de que el plan de producción se respete para que se cumpla el plan de emisiones. Y en cuanto a contenidos, debe comprobar que nada compromete la línea editorial de la cadena" (entrevista a Ramón González, 14/06/08).

El equipo de producción de la empresa televisiva se completa con un productor, que gestiona los recursos y la parte del presupuesto que le compete al canal, y del que dependen los ayudantes y los auxiliares de producción en el que caso de que los haya. La cantidad de componentes de este equipo dependerá del grado de implicación de la cadena en el proceso de producción y de la envergadura del proyecto. De esta forma, cuando se trate de una producción financiada o llave en mano, basta con que un productor ejecutivo o un productor sirva de nexo con la productora, dado que la cadena se limita a financiar el programa. En estos casos, las funciones de este equipo quedan reducidas a una serie de labores esenciales pero burocráticas tal como apunta Ramón González: "La función del equipo de producción se limita a recibir la cinta, comunicar las necesidades del canal, informar de cuándo se necesita el programa, cuánto tiene que durar, cuántos cortes tiene que llevar, cuánto duran los cortes y comprobar qué es lo que entregan, tanto en lo referido a los contenidos como calidad y parámetros técnicos de la imagen" (entrevista a Ramón González, 14/06/08).

Ahondaremos más en estos perfiles profesionales seguidamente, cuando analicemos las funciones de estas figuras en el equipo de producción de la compañía productora, dado que en el caso de las producciones asociadas, este personal se duplica.

\subsection{La jerarquía de producción en la productora}

Del mismo modo que no todas las empresas de televisión poseen una misma estructura organizativa, también encontramos diferencias en las jerarquías de 


\section{El productor ejecutivo de programas de entretenimiento. Jerarquías en la producción audiovisual}

producción que gobiernan las productoras. No obstante, la propuesta presentada en el gráfico 1 sirve de modelo para las principales productoras de la industria audiovisual española, dado que consiste en un organigrama básico que contiene las piezas esenciales implicadas en el proceso de producción de programas.

Aunque a lo largo del apartado anterior ya hemos descrito las principales funciones del productor ejecutivo dependiente de la cadena, tiene mucho más interés analizar esta figura desde la perspectiva de la productora, sobre la que recae en mayor medida el peso creativo de la producción.

En la cúpula de la organización jerárquica de las empresas especializadas en producción, se encuentra el director del área de programas de entretenimiento, máximo responsable de la productora en la puesta en marcha de proyectos de este tipo, tanto desde la vertiente creativa como económica, y que asume la producción ejecutiva de los programas. Las funciones derivadas de esta responsabilidad pueden ser compartidas con otro productor ejecutivo bajo su mando.

Como ya se ha señalado, el productor ejecutivo es el máximo responsable del proyecto, motivo por el que está presente desde su inicio, controlando y supervisando todo el proceso. Como afirma Diego, su principal cometido consiste en "entregar un producto (serie o programa) con un determinado nivel de calidad, ajustándose al presupuesto acordado y en un tiempo previsto" (Diego González, 2005: 11).

Sáinz sintetiza sus funciones del siguiente modo:

El productor ejecutivo (executive producer) o director de producción se encarga del planteamiento, desarrollo, coordinación y control de los productos audiovisuales. Además promueve la cooperación, reúne equipos humanos y especifica las necesidades técnicas de cada proyecto. Es responsable de establecer las fórmulas adecuadas para conseguir la financiación necesaria, busca patrocinios y articula las vías para una eficaz comercialización de los productos que fabrica (Sáinz Sánchez, 1999: 19-20).

El autor emplea indistintamente las denominaciones productor ejecutivo y director de producción, aunque de modo preferente usa el primer término. Incluso, en un texto anterior, hace referencia a los conceptos de jefe de producción o productor jefe para referirse al mismo cargo (Sáinz Sánchez, 1990: 33). No obstante, como se explica en este mismo epígrafe, hemos optado por distinguir, precisamente, entre productor ejecutivo, director de producción y productor (jefe) como perfiles profesionales diferentes.

Siguiendo a Sáinz, el productor ejecutivo no sólo es responsable del equipo de producción, sino también del de realización (1999: 19-20). Más aún, incluso dependen de él los miembros del equipo de dirección. Tanto el productor como 
el director de los programas son profesionales contratados, en última instancia, por el productor ejecutivo.

Su importancia también es destacada por Barroso, para quien, en un sistema de producción en cadena, es necesaria "una figura coordinadora, responsable del producto final y capaz de imprimir una huella o marca propia y peculiar" y que cumpla una triple función (Barroso García, 2002: 36):

1. Creación del programa y control de su evolución.

2. Mantenimiento de las relaciones comerciales con la cadena.

3. Estructuración de un sistema de producción adecuado a las condiciones de la productora.

Millerson, cuyo manual de producción y realización televisiva ha sido un punto de referencia para autores de todo el mundo desde su primera publicación en los años 60 , considera al productor ejecutivo como el máximo dirigente del equipo de producción, aunque le otorga un perfil más administrativo:

Ostenta la jefatura global administrativa y de organización de un grupo de producción (por ejemplo, una serie de programas sobre un tema específico). Él o ella tienen a su cargo el control y la coordinación con la dirección comercial, incluyendo el presupuesto del programa y pueden también estar implicados en temas de mayor amplitud, tales como los arreglos de financiación / patrocinio / coproducción (Millerson, 1991: 412).

No obstante, lo cierto es que en la industria española, a pesar de la extendida visión del productor como un mero burócrata, los productores ejecutivos afirman tener un control absoluto sobre los programas, incluidos los aspectos creativos. No en vano, algunos de los entrevistados para este trabajo proceden de la dirección de contenidos, como es el caso de Daniel Acuña (Videomedia), quien se muestra tajante en este sentido, asegurando que "en un programa de televisión, el productor ejecutivo tiene todo el poder", mediante un control absoluto sobre el proceso: "Básicamente, el productor ejecutivo controla toda la producción del programa. Al inicio de la producción, de él depende la contratación del director, que debe ser de absoluta confianza" (entrevista a Daniel Acuña, 13/06/08).

Para Manuel Aguilera (Grundy), el productor ejecutivo forma parte del equipo desde el inicio y cumple ciertas funciones nucleares para el correcto desarrollo del trabajo:

El productor ejecutivo está presente desde la génesis del formato y es quien mantiene las relaciones con la cadena. Una vez se cierra el acuerdo de producción, sobre contenidos, número de emisiones y otros aspectos, el productor ejecutivo es el 


\section{El productor ejecutivo de programas de entretenimiento. Jerarquías en la producción audiovisual \\ responsable de contratar al personal. La formación de los equipos y la relación con la cadena son dos funciones básicas que nunca deben cederse al director, porque pueden generarse problemas (entrevista a Manuel Aguilera, 17/06/08).}

Precisamente, con el objetivo de centrarse en la supervisión creativa de los programas, el productor ejecutivo delega el control económico en la figura del director de producción, que actúa como vínculo con el departamento contable de la productora (gráfico 1). Daniel Acuña explica la relación entre ambos profesionales en Videomedia:

\footnotetext{
Yo soy director de programas de entretenimiento, así que ejerzo como productor ejecutivo en los distintos programas. Bajo mi supervisión se encuentra la directora de producción, quien se ocupa de la gestión económica, elaborando los presupuestos y llevando un control presupuestario de cada programa. Además, para cada espacio, contrata a un responsable de producción, que depende de ella (entrevista a Daniel Acuña, 13/06/08).
}

Además de la gestión económica de los proyectos, Víctor Martín (Globomedia) otorga al director de producción la labor de diseñar la fórmula idónea de trabajo para cada programa: "El director de producción define las estrategias de producción, el plan de producción. Lo más importante es establecer el planteamiento de la fórmula de trabajo, que es básica, sobre todo en el arranque" (entrevista a Víctor Martín, 16/06/08).

Este profesional, que ha ejercido tanto de productor ejecutivo como de director de producción en Globomedia, destaca que la principal diferencia entre ambos cargos reside en el perfil más creativo del productor ejecutivo:

El productor ejecutivo es un director en excelencia, es el que tiene que definir la línea de contenido que debe seguir el programa, cómo hay que proceder y los plazos para su consecución. Sin embargo, el director de producción se dedica, exclusivamente, a controlar los gastos, los tiempos y las fórmulas de trabajo para obtener un resultado de calidad (entrevista a Víctor Martín, 16/06/08).

Bardají y Gómez explican que la dirección de producción "se encarga de los temas referentes a la contratación, el presupuesto, planificación y seguimiento de los programas" y añaden que "los productores de los programas dependen de esta dirección, que coordina su labor" (2004: 138).

El responsable de la producción diaria de los programas es el productor, quien, a grandes rasgos, desarrolla tres tareas principales: a) gestión económica, b) planificación del trabajo y c) contratación (Sáinz Sánchez, 1999, 36). Fernández y Martínez consideran al productor como una figura esencial para lograr la eficiencia en la producción mediante el desempeño de sus funciones: 
El productor lleva en televisión el seguimiento presupuestario del programa. Su responsabilidad es que personal y medios se combinen en el tiempo pronosticado, realizando, para ello, todas las tareas previstas y con los resultados esperados. Sobre este personal descarga el peso y la coordinación del equipo de producción en su vertiente más operativa (1994: 82).

Sáinz coincide en otorgar al productor un perfil preferentemente administrativo y logístico: "El puesto de trabajo de productor consiste básicamente en la realización de las tareas de coordinación y planificación, presupuestación, grabación y postproducción para la elaboración de un programa o serie de ellos, gestiona y facilita los recursos humanos y técnicos necesarios para la consecución de los objetivos marcados" (1995:48).

Este mismo autor apunta al productor como el responsable de la gestión y control de todos los pagos de una producción, dado que es quien da el visto bueno a todos los gastos y quien liquida periódicamente las cuentas, tareas para las que puede contar con el apoyo de personal contable o administrativo. Además, considera que, en el desempeño de su tarea de planificación, debe idear un sistema de producción seriada que permita la estandarización del trabajo: en programas de emisión regular (semanal o diaria) configura la organización básica que permite estandarizar las tareas dando continuidad a la emisión. Es frecuente que el productor asistido por sus ayudantes, se encargue del desglose, localizaciones, plan de trabajo y presupuesto (Sáinz Sánchez, 1995:49).

Víctor Martín (Globomedia) denomina a esta figura jefe de producción y considera que es "quien planifica el plan de grabación y el trabajo de los equipos bajo la supervisión del director de producción" (entrevista a Víctor Martín, 16/06/08).

Para acometer estas funciones, el productor cuenta con el apoyo de uno o varios ayudantes de producción y de auxiliares, cuyo número puede variar según la complejidad del proyecto. Sáinz describe ampliamente las funciones de este profesional, quien "coopera con el productor en todas las tareas que no impliquen la toma de decisiones estratégicas sobre la planificación y el presupuesto" y que "tiene autonomía para determinar las necesidades de cada grabación", pudiendo sustituir al productor cuando lo requiera la gestión de la producción. Este autor resume en los siguientes puntos sus labores principales (1999: 36-37):

1. Gestión de producción: Obtiene información sobre los medios técnicos, artísticos y humanos, asegurando su disponibilidad en el tiempo y buscando alternativas para adecuar calidad y coste, optimizando el gasto y generando economías de escala. En ausencia del productor, es responsable de la gestión diaria y, consecuentemente, planifica y controla las tareas que desarrollan el resto de equipos. En los viajes se encarga de la intendencia, manutención y hospedaje del equipo humano. Establece calendarios y citaciones, y distribuye la información sobre el plan de trabajo. 
2. Regiduría: Es el responsable del desarrollo, gestión y mantenimiento de las bases de datos de producción. Realiza el desglose y las peticiones correspondientes vinculadas a la dirección artística y escenográfica del programa, ocupándose además de su custodia. Además, reserva y alquila equipo de grabación, supervisa su empleo y devuelve los recursos empleados, encargándose de gestionar su pago.

3. Administración: Al encargarse de la logística, desarrolla actividades administrativas de acuerdo con las normativas de la empresa productora. En este sentido, se ocupa de una amplia variedad de tareas: clasificación, registro y archivo de materiales, preparación de informes y presupuestos, actualización de la agenda de producción, preparación de contratos artísticos, planificación de itinerarios y desplazamientos, firma de partes, etc. Dado que muchas de sus labores de producción están vinculadas a la gestión económica, el ayudante maneja dinero y debe justificar documentalmente los pagos realizados mediante las oportunas liquidaciones.

4. Transportes: Planifica todos los aspectos relacionados con el transporte de materiales y personal.

Además de las categorías profesionales destacadas, todas ellas pertenecientes al área de producción, el equipo encargado de la realización de un programa de televisión también lo integran otros miembros, que son destacados por Fernández y Martínez:

El equipo que compone un programa de televisión lo integra el director o responsable de los contenidos sin competencia en técnica y lenguaje audiovisual que, a veces, es el conductor o la estrella del mismo, los guionistas, redactores y documentalistas para la confección de los guiones, el equipo de realización compuesto por el realizador y sus ayudantes (uno de ellos el regidor de plató), el productor, ayudantes y auxiliares de producción que gestionan los medios, los técnicos, el personal de atrezzo, actores, colaboradores, animadores y público asistente a las grabaciones (1994:83).

El trabajo coordinado de todos los equipos, liderados por el productor ejecutivo, se revela esencial para el buen desarrollo del proyecto.

\section{CONCLUSIONES}

Desde los orígenes de la televisión en España, siempre ha existido una figura creativa principal que ha ejercido el liderazgo en el proceso de producción de programas. Durante las primeras décadas de TVE, el realizador-autor destacó como el máximo responsable, un profesional de un perfil exclusivamente artístico-creativo. No obstante, en los años noventa, con la consolidación de la producción televisiva como industria y como negocio, se comenzó a considerar al 
productor ejecutivo como el máximo responsable de los proyectos, tanto desde una perspectiva económica y logística, como creativa. Las principales características que definen su perfil profesional son: creatividad, capacidad de liderazgo, polivalencia, talento, amplios conocimientos de las diversas áreas de la industria televisiva, experiencia previa, intuición, autoridad, serenidad y capacidad de gestión, organización y coordinación.

La toma de decisiones referida a la producción audiovisual sigue una jerarquía en cuya cúspide se encuentra el productor ejecutivo. En el caso de la modalidad de producción más compleja, la asociada, el equipo de producción de los programas se duplica, encargándose uno de las competencias que corresponden a la cadena, y el otro de las funciones de la productora. Ambos equipos gestionan sus propios presupuestos y sus responsabilidades varían según el acuerdo pactado en el contrato de producción. Tanto el equipo de la productora como el de la cadena suelen componerse de los siguientes integrantes: productor ejecutivo (máximo responsable del proyecto); productor (encargado de organizar la producción de campo del programa y de su control económico y logístico); ayudantes de producción; auxiliares de producción; y personal contable o administrativo.

Del análisis de la estructura organizativa de las empresas audiovisuales, se desprende que el productor ejecutivo de la productora representa la máxima autoridad tanto en cuestiones creativas como económicas, aunque el control de este segundo aspecto puede cederlo en un director de producción de perfil administrativo, para centrase exclusivamente en los aspectos creativos. Sin embargo, dado que el control de la producción lo ostenta la cadena, como agente que financia el proyecto, su productor ejecutivo tiene la capacidad de tomar la última decisión en todo tipo de asuntos.

Aunque la producción ejecutiva controla la línea general del contenido de los programas de entretenimiento, la dirección de los contenidos concretos de cada emisión corresponde al director y su equipo, así como la puesta en imagen es competencia del área de realización. Las decisiones creativas de ambos equipos son supervisadas por el productor ejecutivo, puesto que todas ellas implican un coste económico.

En resumen, puede afirmarse que la industria audiovisual, y la televisiva en particular, se basa en el desarrollo de proyectos "fabricados" en serie (project-based), liderados por un productor ejecutivo (project manager). Desde este punto de vista, la producción ejecutiva de programas de entretenimiento se entiende como una actividad cuyo núcleo lo constituye la dirección de proyectos (project management), área desde la que se supervisa todo el proceso. 


\section{El productor ejecutivo de programas de entretenimiento. Jerarquías en la producción audiovisual \\ REFERENCIAS BIBLIOGRÁFICAS}

AZURMENDI ADARRAGA, A. (2011): Derecho de la comunicación. Barcelona: Bosch. BARDAJÍ HERNANDO, J. y GÓMEZ AMIGO, S. (2004): La gestión de la creatividad en televisión. El caso de Globo Media, Pamplona: Eunsa.

BARROSO GARCÍA, J. (2002), Realización de los géneros televisivos, Madrid: Editorial Síntesis.

BLUMENTHAL, H. J. y GOODENOUGH, O. R. (1991): This business of television. Nueva York: Billboard Books.

BUSTAMANTE, E. (1999): La televisión económica. Financiación, estrategias y mercados, Barcelona: Gedisa Editorial.

CLEMENTS, S. (2004): Show Runner: Producing Variety and Talk Shows for Television. Los Angeles: Silman-James Press.

CONTRERAS, J. M. y PALACIO, M. (2001): La programación de televisión. Madrid: Editorial Síntesis.

DE AGUILERA GAMONEDA, J. (1965): La realización en televisión. Madrid: Imprenta del Servicio de Publicaciones del Ministerio de Información y Turismo.

DIEGO GONZÁLEZ, P. (2005): "La figura del productor de ficción en televisión". Comunicación y Sociedad, 18, junio, pp. 9-30.

ÉCIJA BERNAL, H. (2000), Libro blanco del audiovisual. Cómo producir, distribuir y financiar una obra audiovisual. Madrid: Écija \& Asociados Abogados.

FERNÁNDEZ DÍEZ, F. y MARTÍNEZ ABADÍA, J. (1994): La dirección de producción para cine y televisión. Barcelona: Paidós.

FERNÁDEZ-QUIJADA, D. (2009): "El mercado de la producción independiente en España ante la aparición de Cuatro y laSexta". Comunicación y Sociedad, 22, junio, pp. 59-88.

-(2009): "Producción independiente de televisión en Andalucía. Aproximación a la estructura de un sector desconocido". Ámbitos, 18, pp. 191-209

GUERRERO, E. (2010): El entretenimiento en la televisión española. Historia, industria y mercado. Barcelona: Ediciones Deusto.

HART, C. (1999): Television program making: everything you need to know to get started. Oxford: Focal Press.

HERRERO SUBÍAS, M. y DIEGO GONZÁLEZ, P. (2009): "Series familiares de televisión: concepto, producción y exportación”. Revista Latina de Comunicación Social, 64, pp. 238247.

KELLISON, C. (2009): Producing for TV and New Media. A Real-World Approach for producers. Amsterdam, Boston: Elsevier/Focal Press.

Ley 23/2006, de 7 de julio, por la que se modifica el texto refundido de la Ley de propiedad intelectual, aprobado por el Real Decreto Legislativo 1/1996, de 12 de abril.

Ley 55/2007, de 28 de diciembre, del cine.

LUENGO CRUZ, M. (2008): "El producto cultural: claves epistemológicas de su estudio". Zer, 24, pp. 317-335.

MEDINA LAVERÓN, M. (1998): Valoración publicitaria de los programas de televisión. Pamplona: Eunsa.

MILLERSON, G. (1961): The technique of television production. Londres: Focal Press. -(1983): Effective TV production. Londres: Focal Press. 
-(1991): Técnicas de realización y producción en televisión. Madrid: Instituto Oficial de Radio y Televisión.

-(1999): Television production. Oxford: Focal Press.

-(2009): Realización y producción en televisión. Barcelona: Omega.

NEWCOMB, H. y ALLEY, R. S. (1983): The producer's medium. Conversations with creators of american TV. Oxford: Oxford University Press.

PARDO, A. (2002): The audiovisual management handbook. An in-dephth look at the film, television and multimedia industry in Europe. Madrid: Media Business.

SÁINZ SÁNCHEZ, M. (1990): Iniciación a la producción en televisión. Madrid: Instituto Oficial de Radio y Televisión.

-(1995): Manual básico de producción en televisión. Madrid: Instituto Oficial de Radio y Televisión.

-(1999): El productor audiovisual. Madrid: Editorial Síntesis.

SCHIHL, R. J. (1992): Talk show and entertainment program: processes and procedures. Boston: Focal Press.

ZETTL, H. (2006): Television Production Handbook. Belmont: Thomson Wadsworth.

\section{Cuestionarios}

Productores ejecutivos de cadenas españolas (2008): TVE 1, Tele 5 y Antena 3.

Productores ejecutivos de productoras españolas (2008): Gestmusic-Endemol, El Terrat, Globomedia, Cuarzo, Europroducciones, Videomedia, Martingala, Grundy y Notro TV.

\section{Entrevistas}

Entrevista a Víctor Martín, productor ejecutivo de programas de entretenimiento de Globomedia, 16/06/08, Madrid.

Entrevista a Daniel Bilbao, presidente de Martingala TV y productor ejecutivo de programas de entretenimiento, 17/06/08, Madrid.

Entrevista a José Jesús Lara, director de la división de producciones autonómicas y productor ejecutivo de Europroducciones, 16/06/08, Madrid.

Entrevista a Daniel Acuña, director de la división de programas de entretenimiento de Videomedia, 13/06/08, Madrid.

Entrevista a Ramón González, ex-productor ejecutivo de Tele 5 y ex-subdirector de programas de Telemadrid, 14/06/08, Madrid.

Entrevista a Manuel Aguilera, ex-director de producción de programas de entretenimiento de Grundy, 17/06/08, Madrid.

Entrevista a Jorge Ventosa, director de producción de programas de entretenimiento de 7 y Acción, 25/11/11, Madrid.

\section{Breve semblanza del autor}

Enrique Guerrero se especializó en 2007 en Entertainment and Media Management por la Universidad de California en Los Ángeles (UCLA) y, en 2009, obtuvo el doctorado en 
280 El productor ejecutivo de programas de entretenimiento. Jerarquías

en la producción audiovisual

Comunicación Audiovisual con premio extraordinario por la Universidad de Navarra, donde es profesor de las asignaturas Gestión de contenidos y Producción y guión de entretenimiento. Previamente, había desarrollado su actividad profesional en Telecinco, cadena para la que trabajó en el área de producción de varios programas. Destaca como autor del libro "El entretenimiento en la televisión española. Historia, industria y Mercado" (Deusto, 2010). Además, es miembro de la Academia de las Ciencias y las Artes de Televisión (ATV).

(Recibido el 20-06-2011; aceptado el 02-12-2011) 